CLINICAL STUDY

\title{
Long-term follow-up of prenatally treated children at risk for congenital adrenal hyperplasia: does dexamethasone cause behavioural problems?
}

\author{
Tatja Hirvikoski ${ }^{1,2}$, Anna Nordenström ${ }^{2,3}$, Torun Lindholm ${ }^{5}$, Frank Lindblad ${ }^{6}$, E Martin Ritzén $^{4}$ \\ and Svetlana Lajic ${ }^{2}$ \\ Departments of ${ }^{1}$ Psychiatry, ${ }^{2}$ Molecular Medicine and Surgery, ${ }^{3}$ Clintec, and ${ }^{4}$ Woman and Child Health, Karolinska Institutet, 17176 Stockholm, \\ Sweden, ${ }^{5}$ Department of Psychology and ${ }^{6}$ The Institute for Stress Research, Stockholm University, 10691 Stockholm, Sweden \\ (Correspondence should be addressed to S Lajic who is now at Department of Molecular Medicine and Surgery, Centre for Molecular Medicine L8:02, \\ Karolinska Institutet/Karolinska University Hospital, SE-17176 Stockholm, Sweden; Email: svetlana.lajic@ki.se)
}

\begin{abstract}
Objectives: To investigate the long-term effects of prenatal treatment of congenital adrenal hyperplasia $(\mathrm{CAH})$ with emphasis on behavioural problems and temperament.

Design: A population-based long-term follow-up study of Swedish children at risk for virilising CAH, who had received treatment prenatally with dexamethasone (DEX). The questionnaire-based follow-up was performed when the children had reached school age.

Methods: Standardised parent-completed questionnaires were used to evaluate adaptive functioning, behavioural/emotional problems and psychopathology, social anxiety and temperament in DEXexposed school-aged children $(n=26)$ and matched controls $(n=35)$. In addition, the association between parental questionnaires and children's self-ratings was investigated.

Results: There were no statistically significant differences between DEX-exposed children and controls in measures of psychopathology, behavioural problems and adaptive functioning. In a questionnaire on temperamental traits, DEX-exposed children were described by their parents as being more sociable than controls $(P=0.042)$. The correlation analysis showed only modest parent-child agreement on social anxiety, i.e. the increased social anxiety in children's self-ratings was not confirmed by their parents. Conclusions: DEX-treated children showed good overall adjustment. The parent-child agreement with respect to social anxiety was modest, highlighting the importance of multiple information sources and assessment methods. The clinical significance of the observed difference in sociability cannot be determined within the frameworks of this study. Additional studies of larger cohorts are essential to make more decisive conclusions on the safety of the treatment. Until then, it is important that parents are thoroughly informed about the benefits and potential risks and uncertainties of this controversial treatment.
\end{abstract}

European Journal of Endocrinology 159 309-316

\section{Introduction}

The clinical symptoms of 21-hydroxylase deficiency range from mild disease (non-classical congenital adrenal hyperplasia $(\mathrm{CAH}))$ presenting in women during adolescence or later due to signs of hirsutism or infertility to more severe prenatally virilising forms with or without salt loss (simple virilising $\mathrm{CAH}$, SV and salt-wasting $\mathrm{CAH}$, SW respectively). The newborn girl with virilising $\mathrm{CAH}$ (SV or SW) can, in the most severe cases, be mistaken for a boy due to a persistent urogenital sinus, labioscrotal fusion and clitoromegaly, leading to a traumatising experience for the family. There is a general agreement that these malformations can be reduced or prevented by prenatal treatment with the synthetic glucocorticoid (GC) dexamethasone (DEX) and, consequently, reconstructive surgery can be avoided (1-3). To be fully effective the DEX treatment has to be started in the sixth to seventh postmenstrual week and continued until the results of the prenatal diagnosis by means of a chorionic villous biopsy are available. This means that seven out of eight foetuses (boys and unaffected girls) are treated unnecessarily during early gestation. Girls with $\mathrm{CAH}$ are treated until term. Treatment safety has been reported to be acceptable, at least in the short-term perspective, based on findings of normal pre- and postnatal growth $(1,4-6)$, and the side effects that have been reported do not appear to follow a particular pattern $(4,6-8)$. Thus, the current short-term follow-up studies on humans suggest that DEX treatment does not have major teratogenic effects.

Nevertheless, long-term effects are just starting to be addressed due to the fact that the oldest children treated are now reaching early adulthood. Results from experimental animal models have also raised concerns 
regarding the impact of the treatment on foetal programming and the possible effects on future metabolic and cognitive functions in adult life (9-11). In human foetal development, many limbic and cortical brain structures as well as neural layers develop during early gestation, i.e. before the results of chorionic villous sampling are available (12). For the CAH-affected girls, possible negative consequences, if minor, may be balanced against positive effects of preventing virilisation of external genitalia. Nevertheless, only one out of eight treated children is a CAH-affected girl. Even with early foetal sex typing, using PCR amplification of cell-free foetal DNA in maternal blood, performed during the first trimester (1315), the ethical dilemma remains that healthy foetuses (seven out of eight versus three out of eight) will be treated for a short period of time for the sake of benefit of others.

Experimental data from animals exposed to prenatal corticosteroids have shown adverse effects on somatic development and cognitive functions, as well as behaviour. In rats, a range of adverse effects has been observed upon prenatal GC treatment: low birth weight and hypertension (16), decreased size of the hippocampus and affected short-term memory (9) and alterations in structural brain development as well as in different neurotransmitter systems (17-19). Behavioural inhibition has also been observed in rats exposed to DEX during the entire gestational period or during late gestation (20). However, animal experiments aimed at identifying possible risks with prenatal GC treatment have primarily been designed to mimic the treatment used to reduce the risk of respiratory distress syndrome in preterm infants, and GCs have thus been given in late gestation. Nonetheless, these studies may be relevant in the view of the fact that the developmental timetable of the foetus differs significantly in rats and humans and many areas of the brain that develop in the rat from mid- to late gestation are formed in humans during the first trimester of pregnancy (12). Rodent and primates differ in GC receptor distribution in the brain and the corticosensitivity (21-23). In addition, in rhesus monkeys, damage on hippocampal neurons has been observed after prenatal DEX exposure in late pregnancy (24). There is sparse clinical evidence pertaining to the effects of low-dose prenatal DEX treatment as used in CAH. It should be noted that behavioural effects, including cognition, are not necessarily mediated by structural changes in the brain, since GCs are essential for the modulation of behavioural adaptation during acute stress and regulate arousal, alertness and cognition $(25,26)$. Prenatal administration of DEX to African vervet monkeys was associated with metabolic effects as well as an exaggerated cortisol response to mild stress (27).

Few follow-up studies on the developmental outcome of DEX-exposed children have been conducted and only one, performed by our group, has been based on direct assessments of the treated children (28). A pilot study, designed as a postal maternal questionnaire survey and comprising 26 long- and short-term-treated pre-school children (mean age $=2.5 \pm 1.3$ years), suggested that DEX-exposed children showed more shyness, greater emotionality, less sociability and more internalising problems (29). A questionnaire study conducted by the same researchers on a larger sample (30) focused on parent-reported cognitive and motor development and did not detect any significant differences. In the case of pre-school children, general developmental scales were completed by the parents while school scale data from the Child Behaviour Check List (CBCL) were available for the children aged 6-12. Data on the children's temperament or behavioural problems were not presented.

We have previously reported results from direct neuropsychological assessments and self-rating questionnaires of school-age children and adolescents treated with DEX (28). No differences in major cognitive measures such as IQ, learning and long-term memory were found. However, prenatal DEX treatment was associated with negative effects on the verbal working memory and children's perceptions of their scholastic competence and social anxiety. These effects were observed in short-term-treated, CAH-unaffected children, compared with healthy controls. The CAH-affected children, treated with hydrocortisone postnatally, did not differ significantly from the controls, probably due to the small group size.

In the present study, the long-term emotional and behavioural development were assessed by parental questionnaires. As this is the first study assessing parent-rated temperament, behavioural problems and psychopathology in school-aged DEX-treated children and adolescents, the design was explorative with a broad range of investigated variables. Measures of general psychopathology and adaptive functioning were included. Based on previous results in pre-school children (29), we also investigated the prevalence of shyness, social anxiety and inhibition.

\section{Methods}

\section{Study subjects}

In Sweden, mothers who have given birth to a child with a severe form of CAH (as determined by CYP21 genotype) are offered DEX-treatment (at a dose of $20 \mu \mathrm{g} / \mathrm{kg}$ per day in three divided doses based on pre-pregnancy weight and a maximum of $1.5 \mathrm{mg}$ daily) following pregnancies in order to prevent prenatal virilisation of affected female foetuses. During the years 1985-1995, 40 foetuses at risk of being affected with a severe form of $\mathrm{CAH}$ were treated with DEX from gestational weeks $6-7$ (6). In this retrospective study, families with a DEX-treated child were contacted by letter. None of the families had been involved initially within the framework of a clinical study. The final cohort comprised 26 children (12 boys, 14 girls; refusal rate $35 \%$ ) between 7 and 17 years of age (mean \pm 
S.D., $10.95 \pm 2.33$ years). Based on the results of a mutation analysis performed on DNA from chorionic villous biopsies sampled at week 10-12, treatment was either continued to term (four CAH-affected females) or stopped at the end of the first trimester (seven unaffected boys, ten unaffected girls and five CAH-affected boys). In order to investigate whether the group of children and families that participated in the study differed from the group that refused participation, a short-structured interview was given to the paediatrician responsible for the clinical follow-up of the older CAH-affected sibling. The groups did not differ with regard to psychosocial problems in the family. Furthermore, the group that participated in the study did not differ from the group that refused to participate with respect to maternal side effects induced by DEX and the maternal attitude to future treatment, as assessed by maternal questionnaires.

Two control groups were recruited. First, siblings to children treated with growth hormone (with psychosocial prerequisites presumed to be similar to those of the study group, i.e. an older sibling with a chronic illness) were included: 10 (four boys and six girls) $/ 28$ children (refusal rate 64\%) between 7 and 12.5 years of age $(9.94 \pm 2.02)$. Secondly, in order to create a large enough control group, the Swedish National Registry was used to randomly recruit 25 children matched for age and sex ( 11 boys and 14 girls, refusal rate $69 \%$ ) between 7 and 17 years of age $(10.55 \pm 2.46)$.

Among the DEX-treated children, 54\% lived in cities in comparison with $97 \%$ of the controls $\left(\chi^{2} P<0.001\right)$. There were no significant differences in parental socioeconomic status (SES) as estimated by the parental educational level $\left(\chi^{2} P<0.162\right)$ between the two groups. Moreover, the treated versus non-treated children were comparable regarding birth length $(P=0.234)$, birth weight $(P=0.704)$ and gestational length $(P=0.942)$.

\section{Study design}

Parental questionnaires were administered in a standardised manner and were completed during a visit to the clinical psychologist. Consequently, the parents had the opportunity to request clarification of the items as recommended in the manual for the CBCL (31). The results of the parental questionnaires were compared with the previously published results of the children's self-rating questionnaire (28). Parents and children completed the questionnaires independently of each other, and without any influence of the clinical psychologist. All families gave their written informed consent, and the study was approved by the Regional Ethics Committee of Stockholm.

\section{Instruments}

Behavioural problems/psychopathology The CBCL for ages 4-18 (CBCL/4-18) (31) is a frequently used 113-item child psychiatric screening instrument.
It provides broadband (internalising and externalising) and narrowband (withdrawn, somatic complaints, anxious/depressed, social problems, thought problems, attention problems, delinquent behaviour and aggressive behaviour) scales. One-week test-retest reliabilities for the internalising, externalising and total problem scales ranged from 0.89 to 0.93 , and Cronbach's $\alpha$ (i.e. the internal consistency) ranged from 0.89 to 0.96 . The Social Phobia and Anxiety Inventory for Children (SPAIC) (32) was initially developed as a 26-item self-report measure of cognitive, physiological and behavioural symptoms of social phobia according to the Diagnostic and Statistical Manual of Mental Disorders (DSM-IV) (33). Using factor analysis, both three- and five-factor solutions have been identified $(32,34)$. The SPAI-C has demonstrated a good 2-week test-retest reliability $(r=0.86)$ and internal consistency (Cronbach's $\alpha=0.95$; Beidel et al. 1995). A confirmatory factor analysis of SPAI-C, designed as a parental questionnaire (SPAI-C-P, SPAI-C - Parent Report) (35), supported the three-factor model over the five-factor model. The three factors are 1) assertiveness/general conversation; 2) traditional social encounters and 3) public performance. The overall Cronbach's $\alpha$ for SPAI-C-P was 0.93, and the scale demonstrated both concurrent and discriminant validity (35).

Competence profile/adaptive functioning The CBCL total competence score was used to measure adaptive functioning. The total competence score is the sum of the raw scale scores obtained from three subscales: 1) activities scale - the number, amount and quality of the child's participation in the various activities; 2) social scale - participation in organisations, the number of both close friends and weekly contacts with them, how well the child gets along with others as well as how well the child plays and works alone and 3) school scale performance in academic subjects, receipt of special remedial services and school problems. The internal consistency of the total competence scale ranged from 0.57 to 0.64 , and the 1 -week test-retest reliability was 0.87 (31).

Temperament The Emotionality-Activity-SociabilityShyness Temperament Survey for Children (EAS) (36)) was used to assess the children's temperament. The questionnaire describes four dimensions of temperament that are measured by subscales: 1) emotionality - the tendency to become aroused easily and intensely; 2) activity - preferred levels of activity and speed of action; 3) shyness - the tendency to be inhibited and awkward in new social situations and 4) sociability - the tendency to prefer the presence of others to being alone. Cronbach's $\alpha$ for the first three scales averaged 0.83 and the 1 -week test-retest reliability ranged between 0.58 and 0.80 . The sociability scale had not yet been subjected to all psychometric tests (36). 


\section{Statistical analysis}

Similar results were obtained for the two control groups on all measures and therefore data for the two groups were combined. Most of the variables were positively skewed (i.e. many low ratings) and especially ratings on the CBCL scales were radically skewed. Therefore, nonparametric statistics was applied for between-group comparisons. The DEX-treated group consisted both of $\mathrm{CAH}$-unaffected children and $\mathrm{CAH}-$ affected patients. DEX-exposed children were compared with unexposed children using the Mann-Whitney $U$ test. However, statistically significant findings were re-evaluated using the Kruskall-Wallis test for comparisons of three groups: DEX-treated CAH-unaffected children, DEXtreated CAH-affected children and controls. In the DEX-treated CAH-affected group, both full-term-treated girls and short-term-treated CAH-affected boys were included (i.e. all children that had received GC both preand postnatally), thus enabling comparison of CAHunaffected children with controls while excluding the influence of postnatal GC treatment.

The effect size for between-group differences was calculated for the Mann-Whitney $U$ statistic using the method described by Newcombe, $2006(37,38)$. This relative effect size statistic, $\theta$, can be regarded as expressing the degree of separation between two frequency distributions. The statistic $\theta$ is analogous to the standardised difference obtained for normally distributed data by dividing the difference of the means by the pooled standard deviation $(37,38)$.

In order to examine the parent-child agreement on measures of social anxiety, we correlated the different measures with each other. Although Kendall's $\tau$ coefficient may be chosen for non-normal data, this may be necessary only at quite extreme levels, which was not the case with regard to the measures of social anxiety. Pearson's correlation coefficient is generally used for analysis of raw data and was chosen in this study (39 p. 288). The results of children's self-ratings have been reported previously (28).

The study is explorative and includes many statistical comparisons. A Bonferroni correction across all outcome variables would, however, lead to a significant loss of power in small groups such as those presented here, which means that the risk of type II errors would increase considerably. Therefore, uncorrected $P$ values are reported. Although only negative effects, if any at all, were expected for the treated children, two-tailed statistical tests were chosen in order to reduce the risk of type I errors. The $\alpha$ level was set to $P=0.05$.

\section{Results}

\section{Behaviour problems/psychopathology}

No significant differences between DEX-treated children and the control group were observed with respect to parental ratings of behavioural problems or psychopathology using the eight CBCL syndrome scales (data not shown). The CBCL data are summarised on the level of the two broad groupings of syndromes, i.e. internalising and externalising problems, as well as the total problem score (Table 1). None of the three factors of the SPAI-C-P report demonstrated any differences between the DEX-exposed children and the control group (Table 1).

Table 1 Results of the parent-completed questionnaires: Child Behaviour Check List (CBCL), Social Phobia and Anxiety Inventory for Children-Parental Ratings (SPAI-C-P) and Emotionality-Activity-Sociability-Shyness Temperament Survey for Children (EAS).

\begin{tabular}{|c|c|c|c|}
\hline & $\begin{array}{l}\text { DEX-treated group }(n=26) \\
\text { Median (range) }\end{array}$ & $\begin{array}{l}\text { Control group }(n=35) \\
\text { Median (range) }\end{array}$ & Mann-Whitney $U$ test \\
\hline \multicolumn{4}{|l|}{ CBCL } \\
\hline Total competence scale & $16.16(7.50-25.12)$ & $17.75(11.73-23.83)$ & $U=348, P=0.119$ \\
\hline Internalising problems & $4.00(0-17)$ & $5.00(0-25)$ & $U=387, P=0.319$ \\
\hline Externalising problems & $4.50(0-34)$ & $5.00(0-21)$ & $U=446, P=0.895$ \\
\hline Total problems & $17.00(0-64)$ & $17.00(2-75)$ & $U=447, P=0.907$ \\
\hline \multicolumn{4}{|l|}{ SPAI-C-P } \\
\hline Assertiveness/general conversation & $3.50(0-16)$ & $5.00(0-9)$ & $U=386, P=0.315$ \\
\hline Traditional social encounters & $0.00(0-9)$ & $1.00(0-4)$ & $U=396, P=0.356$ \\
\hline Public performance & $3.00(0-14)$ & $2.00(0-10)$ & $U=425, P=0.658$ \\
\hline Total & $6.00(0-42)$ & $8.00(0-23)$ & $U=379, P=0.266$ \\
\hline \multicolumn{4}{|l|}{ EAS Temperament Survey } \\
\hline Activity & $3.40(2.20-5)$ & $3.60(1.80-4.80)$ & $U=440.50, P=831$ \\
\hline Shyness & $1.60(1-3.80)$ & $2.00(1-4.20)$ & $U=350, P=0.124$ \\
\hline Emotionality & $2.70(1.80-4.80)$ & $2.80(1.20-5)$ & $U=451.50, P=0.959$ \\
\hline Sociability & $4.00(2.60-4.80)$ & $3.60(2-5)$ & $U=317, P=0.042^{*}$ \\
\hline
\end{tabular}

${ }^{*}$ On the EAS Temperament Survey DEX-exposed children were found to be more sociable $(P=0.042)$ but in the Kruskall-Wallis test with the three groups (CAH-affected, $\mathrm{CAH}$-unaffected and controls), this difference was no longer significant $(P=0.057)$. The items on the EAS Sociability scale pertain to: likes to be with other people/prefers playing with others rather than alone/finds people more stimulating than anything else/something of a loner (reversed item)/when alone, feels isolated. 


\section{Competence profile/adaptive functioning}

The DEX-exposed children and the controls got comparable results in the parental ratings on the CBCL total competence scale (Table 1).

\section{Temperament}

While results were comparable for the two groups on most EAS subscales (Table 1), parents reported that the DEXexposed children were more sociable than controls $(P=0.042)$ on the EAS sociability scale. The difference between the groups had an effect size $\theta=0.55$. A further comparison of the CAH-affected, CAH-unaffected and control cases using the Kruskall-Wallis test no longer showed significant differences between the groups $(P=0.057)$.

\section{Parent-children agreement on social anxiety}

The correlation was strong between the two parental questionnaires, EAS-Shyness assessing shyness as a temperamental trait and SPAI-C-P assessing social anxiety according to DSM-IV. The children's self-rating on social anxiety, Social Anxiety Scale for ChildrenRevised, was moderately correlated with SPAI-C-P and did not correlate at all with EAS-Shyness. The results of the correlation analysis are summarised in Table 2.

\section{Discussion}

We conducted a retrospective population-based long-term follow-up study of prenatal DEX treatment of children at risk for CAH, based on parental ratings of the children's temperament and adaptive functioning, as well as occurrences of behavioural problems and psychopathology. Good parent-rated overall adjustment was expected in this study, as we had previously observed normal psychometric intelligence, learning and long-term memory, as well as normal parent-rated school performance in the current cohort (28). Accordingly, no differences in parental ratings of behavioural problems/psychopathology and adaptive functioning were observed between treated and non-treated children.

Table 2 Correlation matrix of measures of social anxiety and shyness.

\begin{tabular}{lll}
\hline & SASC-R & SPAI-C-P \\
\hline SPAI-C-P & $r=0.305^{\mathrm{a}}$ & \\
EAS shyness & $r=0.003$ & $r=0.573^{\mathrm{b}}$ \\
\hline
\end{tabular}

SASC-R is a children's self-rating scale, while the other questionnaires are completed by parents. SASC-R, Social Anxiety Scale for Children-Revised; SPAI-C-P, Social Phobia and Anxiety Inventory for Children-Parent Report; EAS Shyness, Emotionality-Activity-Sociability-Shyness Temperament Survey for Children, Shyness subscale.

${ }^{a}$ Correlation is significant at the 0.05 level (two-tailed).

${ }^{b}$ Correlation is significant at the 0.01 level (two-tailed).
A comparison of results on CBCL in the current study with a large Swedish epidemiological study (40) and the non-referred children's norms in the American CBCL manual (31) showed that both DEX-exposed and control children obtain normal scores. The same was observed when our results from parental questionnaires (CBCL and SPAI-C-P) were compared with those of Higa et al. (35), showing that the results for both the DEXtreated group and the controls were very similar to those of normal school-age children. Taken together, the parental ratings of behavioural problems in the current study showed normal results for both the DEX-treated children and controls.

Thus, our results do not confirm those of Trautman et al. (29) which indicated more internalising problems, shyness and emotionality, as well as less sociability, in DEX-treated pre-school children, as measured by parental questionnaires. On the contrary, we found that DEX-exposed children were rated by their parents as more sociable than controls. However, when comparing the three groups (CAH-affected, CAHunaffected and controls), this difference was no longer significant, probably due to small group sizes. The results on other subscales of the EAS temperament survey were comparable across the groups. Sociability is defined as the tendency to prefer the presence of others to being alone and should not be equated with "nonshyness' since shyness refers mainly to behaviour with strangers, i.e. the tendency to be inhibited and awkward in new social situations (36). Most shy children do not experience tension and distress with persons they know well and shyness, but not sociability, is associated with an increased risk of later development of anxiety disorders (41). The finding of a single significant result among all the non-significant results may raise the question of a possible type I error (i.e. rejecting a true null hypothesis). On the other hand, this finding had an effect size of $\theta=0.55$, indicating a difference that corresponds to half a standard deviation between the two distributions. However, we must consider whether the difference in sociability is due to the DEX treatment per se or if it is an expression for a coping strategy among siblings to children with a chronic disorder i.e. a way of seeking attention from the parents.

The observed similarities between the treated children and controls in parental ratings of social anxiety stand in contrast to differences in the children's self-ratings. We have previously reported that prenatal DEX treatment was associated with increased self-reported social anxiety (28). Parental ratings did not confirm this finding. However, previous studies on cross-informant agreement show that parent-child agreement is only modest (42). Given that parents and children may focus on different aspects of child psychopathology (43), neither parent's reports nor children's self-ratings should be regarded as invalid or incorrect. Instead, multi-source assessment procedures are recommended $(35,42,43)$. 
We should also consider whether we may have failed to note an existing effect on social anxiety in parental ratings, thus committing a type II error (assuming that the null hypothesis is true when it is not). The high refusal rate in the DEX-treated group, which was most probably due to the fact that DEX treatment was not administered within the framework of a clinical study, may increase the risk of a type II error if children with most problem behaviours do not participate. The schematic analysis of the group that refused to participate versus the group that participated in the study did not show any significant differences between the groups, however. The second factor that may increase the risk of a type II error is the fact that more DEX-treated children lived in rural areas compared with controls and the prevalence of behavioural problems and mental disorders among children and adolescents has been reported to be similar in urban and rural areas (44) or even lower in rural areas (45, 46). This may, at least theoretically, have an impact on our results by 'masking' problems in the treated group. However, in a Swedish study (40), differences on CBCL scores relating to demographic and background variables such as gender, age, SES or living area (urban/rural) have been observed to be small and explain less than $4 \%$ of the variance. Furthermore, we had low statistical power in our analyses because only small effects were expected, while a small sample like ours has only reasonable power to detect a large effect (47). This is especially important when possible effects are measured using questionnaires, many of which are designed to capture clinical problems and may not be sensitive enough to identify subclinical effects. It is important to be aware of the fact that subclinical effects, though subtle, may lead to impairment in everyday life when the child encounters new or challenging tasks and situations. It should also be noted that non-parametric analyses have less statistical power than parametric ones. It may be argued that we could have used parametric Student's $t$-test analyses for comparisons between groups, at least for variables that were not radically skewed (i.e. other than CBCL scales) as the sample size we had would produce a normal sampling distribution of the mean (39 p 170-179). We therefore re-analysed the data with a series of $t$-tests and obtained the same pattern of results (non-significant for all scales except the EAS Sociability scale; data not shown).

Taken together, the risk of a type II error was high in the current study. Hence, a lack of significant findings in this case cannot be used as proof that the treatment is safe. Nonetheless, our results indicate, as expected, that prenatal DEX treatment of CAH does not cause major behavioural problems or produce large effects, which we should have detected with the statistical power we had. Although based on a population of all cases treated in Sweden during a period of 10 years, the sample size was small. In a rare condition such as $\mathrm{CAH}$, a very long time is needed to collect large samples. However, it is not satisfactory just to wait for the results of ongoing prospective studies (3), as it will take a long time before these results are available and meanwhile healthy siblings of children with CAH are exposed to DEX. Larger retrospective studies using multi-source assessment procedures are of great importance. Assuming that there would be several effects of the same size as the difference in sociability observed in this study $(d=0.55$, approx.), a sample size of 52 (in each group) would provide us with a power of 0.80 , i.e. a $20 \%$ chance of a type II error. In the future, the results from several studies can be combined and analysed by meta-analysis.

In conclusion, using parental ratings as an assessment method, prenatal DEX treatment of CAH does not seem to cause major behavioural problems or psychopathology. This result corresponds to the normal findings for all major cognitive measures such as IQ, learning and long-term memory (28). The clinical significance of increased sociability among DEX-treated children cannot be determined within the framework of this study. Additional studies on larger cohorts are essential to allow more decisive conclusions to be drawn concerning the safety of this controversial treatment, because even small effects may be of importance if they lead to impairment in the child's everyday life. Until then, it is important that the parents are thoroughly informed about the benefits of the treatment for the CAH-affected girls, as well as possible negative effects and uncertainties. Prenatal DEX treatment of children at risk for $\mathrm{CAH}$ should only be carried out in centres where proper follow-up of treated cases can be achieved.

\section{Acknowledgements}

This work was supported by the Karolinska Institutet, Stockholm County Council, Sällskapet Barnavård, Stiftelsen Frimurare Barnhuset, Stiftelsen Sven Jerring, the Söderberg Foundation (CMM) and the Ronald McDonald Foundations. We are indebted to all the children and parents involved in this study. We also wish to express our sincere gratitude to our colleagues involved in the long-term care of the patients, and especially Lo Neumeyer, RN, whose support was invaluable in recruiting the control groups.

\section{References}

1 New MI, Carlson A, Obeid J, Marshall I, Cabrera MS, Goseco A, LinSu K, Putnam AS, Wei JQ \& Wilson RC. Prenatal diagnosis for congenital adrenal hyperplasia in 532 pregnancies. Journal of Clinical Endocrinology and Metabolism 2001 86 5651-5657.

2 Forest MG \& Dörr HG. Prenatal therapy in congenital adrenal hyperplasia due to 21-hydroxylase deficiency: retrospective followup study of 253 treated pregnancies in 215 families. Endocrinologist $200313252-259$. 
3 Lajic S, Nordenstrom A, Ritzen EM \& Wedell A. Prenatal treatment of congenital adrenal hyperplasia. European Journal of Endocrinology 2004151 (Supplement 3) U63-U69.

4 Forest MG \& Dörr HG. Prenatal treatment of congenital adrenal hyperplasia $(\mathrm{CAH})$ due to 21-hydroxylase deficiency: European experience in 223 pregnancies at risk. Pediatric Research 1993 $33 \mathrm{~S} 3$.

5 Mercado AB, Wilson RC, Cheng KC, Wei J-Q \& New MI. Prenatal treatment and diagnosis of congenital adrenal hyperplasia owing to steroid 21-hydroxylase deficiency. Journal of Clinical Endocrinology and Metabolism $1995 \mathbf{8 0} 2014-2020$.

6 Lajic S, Wedell A, Bui TH, Ritzen EM \& Holst M. Long-term somatic follow-up of prenatally treated children with congenital adrenal hyperplasia. Journal of Clinical Endocrinology and Metabolism 1998 83 3872-3880.

7 Haan EA, Serjeantson SW, Norman R, Rollond AK, Antonis P, Richards RI \& Penfold JL. Prenatal diagnosis and successful intrauterine treatment of a female fetus with 21-hydroxylase deficiency. Medical Journal of Australia 1992156 132-135.

8 Couper JJ, Hutson JM \& Warne GL. Hydrometrocolpos following prenatal dexamethasone treatment for congenital adrenal hyperplasia (21-hydroxylase deficiency). European Journal of Pediatrics 1993152 9-11.

9 Seckl JR \& Miller WL. How safe is long-term prenatal glucocorticoid treatment? Journal of the American Medical Association 1997 277 1077-1079.

10 Seckl JR \& Meaney MJ. Glucocorticoid programming. Annals of the New York Academy of Sciences 20041032 63-84.

11 Miller WL. Dexamethasone treatment of congenital adrenal hyperplasia in utero: an experimental therapy of unproven safety. Journal of Urology $1999 \mathbf{1 6 2} 537-540$.

12 Bayer SA, Altman J, Russo RJ \& Zhang X. Timetables of neurogenesis in the human brain based on experimentally determined patterns in the rat. Neurotoxicology 1993 14 83-144.

13 Bischoff FZ, Lewis DE \& Simpson JL. Cell-free fetal DNA in maternal blood: kinetics, source and structure. Human Reproduction Update $20051159-67$.

14 Rijnders RJ, van der Schoot CE, Bossers B, de Vroede MA \& Christiaens GC. Fetal sex determination from maternal plasma in pregnancies at risk for congenital adrenal hyperplasia. Obstetrics and Gynecology 200198 374-378.

15 Morel Y, Tardy V, Costa JM, Forest MG \& David M. 21 Hydroxylase deficiency: new strategies emerging from molecular studies. Annales d'Endocrinologie 200364 456-470.

16 Celsi G, Kistner A, Aizman R, Eklof AC, Ceccatelli S, de Santiago A \& Jacobson SH. Prenatal dexamethasone causes oligonephronia, sodium retention, and higher blood pressure in the offspring. Pediatric Research 199844 317-322.

17 Kreider ML, Aldridge JE, Cousins MM, Oliver CA, Seidler FJ \& Slotkin TA. Disruption of rat forebrain development by glucocorticoids: critical perinatal periods for effects on neural cell acquisition and on cell signaling cascades mediating noradrenergic and cholinergic neurotransmitter/neurotrophic responses. Neuropsychopharmacology 200530 1841-1855.

18 Slotkin TA, Kreider ML, Tate CA \& Seidler FJ. Critical prenatal and postnatal periods for persistent effects of dexamethasone on serotonergic and dopaminergic systems. Neuropsychopharmacology 200631 904-911.

19 McArthur S, McHale E \& Gillies GE. The size and distribution of midbrain dopaminergic populations are permanently altered by perinatal glucocorticoid exposure in a sex- region- and timespecific manner. Neuropsychopharmacology 200732 1462-1476.

20 Welberg LA, Seckl JR \& Holmes MC. Prenatal glucocorticoid programming of brain corticosteroid receptors and corticotrophinreleasing hormone: possible implications for behaviour. Neuroscience $2001 \mathbf{1 0 4} 71-79$.

21 Patel PD, Lopez JF, Lyons DM, Burke S, Wallace M \& Schatzberg AF. Glucocorticoid and mineralocorticoid receptor mRNA expression in squirrel monkey brain. Journal of Psychiatric Research 200034 383-392.
22 Sanchez MM, Young LJ, Plotsky PM \& Insel TR. Distribution of corticosteroid receptors in the rhesus brain: relative absence of glucocorticoid receptors in the hippocampal formation. Journal of Neuroscience 200020 4657-4668.

23 Claman HN. How corticosteroids work. Journal of Allergy and Clinical Immunology 197555 145-151.

24 Uno H, Lohmiller L, Thieme C, Kemnitz JW, Engle MJ, Roecker EB \& Farrell PM. Brain damage induced by prenatal exposure to dexamethasone in fetal rhesus macaques. I. Hippocampus. Brain Research. Developmental Brain Research 199053 157-167.

25 Charmandari E, Kino T, Souvatzoglou E \& Chrousos GP. Pediatric stress: hormonal mediators and human development. Hormone Research $200359161-179$.

26 Lupien SJ, Wilkinson CW, Briere S, Menard C, Ng Ying Kin NM \& Nair NP. The modulatory effects of corticosteroids on cognition: studies in young human populations. Psychoneuroendocrinology 200227 401-416.

27 de Vries A, Holmes MC, Heijnis A, Seier JV, Heerden J, Louw J, Wolfe-Coote S, Meaney MJ, Levitt NS \& Seckl JR. Prenatal dexamethasone exposure induces changes in nonhuman primate offspring cardiometabolic and hypothalamic-pituitary-adrenal axis function. Journal of Clinical Investigation $2007 \mathbf{1 1 7}$ 1058-1067.

28 Hirvikoski T, Nordenstrom A, Lindholm T, Lindblad F, Ritzen EM, Wedell A \& Lajic S. Cognitive functions in children at risk for congenital adrenal hyperplasia treated prenatally with dexamethasone. Journal of Clinical Endocrinology and Metabolism 2007 $92542-548$.

29 Trautman PD, Meyer-Bahlburg HF, Postelnek J \& New MI. Effects of early prenatal dexamethasone on the cognitive and behavioral development of young children: results of a pilot study. Psychoneuroendocrinology 199520 439-449.

30 Meyer-Bahlburg HF, Dolezal C, Baker SW, Carlson AD, Obeid JS \& New MI. Cognitive and motor development of children with and without congenital adrenal hyperplasia after early-prenatal dexamethasone. Journal of Clinical Endocrinology and Metabolism $200489610-614$.

31 Achenbach TM. Manual for the Child Behavior Checklist/4-18 and 1991 Profile. Burlington, VT: University of Vermont, Department of Psychiatry, 1991.

32 Beidel DC, Turner SM \& Morris TL. A new inventory to assess social phobia in children: the Social Phobia and Anxiety Inventory for Children. Psychological Assessment 19957 73-79.

33 American-Psychiatric-Association. Diagnostic and Statistical Manual of Mental Disorders, edn 4 (DSM-IV) Washington, DC, USA: American-Psychiatric-Association, 1994.

34 Beidel DC, Turner SM \& Fink CM. Assessment of childhood social phobia: construct, convergent, and discriminative validity of the Social Phobia and Anxiety Inventory for Children (SPAI-C). Psychological Assessment 19963 235-240.

35 Higa CK, Fernandez SN, Nakamura BJ, Chorpita BF \& Daleiden EL. Parental assessment of childhood social phobia: psychometric properties of the Social Phobia and Anxiety Inventory for Children - Parent Report. Journal of Clinical Child and Adolescent Psychology $200635590-597$.

36 Buss AH \& Plomin R. Temperament: Early Developing Personality Traits. Hillsdale, NJ: Lawrence Erlbaum, 1984.

37 Newcombe RG. Confidence intervals for an effect size measure based on the Mann-Whitney statistic. Part 1: general issues and tail-area-based methods. Statistics in Medicine 200625 543-557.

38 Newcombe RG. Confidence intervals for an effect size measure based on the Mann-Whitney statistic. Part 2: asymptotic methods and evaluation. Statistics in Medicine 200625 559-573.

39 Howell DC. Statistical Methods for Psychology (International Student/6th Edition) Belmont, CA: Thomson Wadsworth, 2006/2007.

40 Larsson B \& Frisk M. Social competence and emotional/behaviour problems in 6-16 year-old Swedish school children. European Child and Adolescent Psychiatry 19998 24-33. 
41 Kagan J, Snidman N \& Arcus D. The role of temperament in social development. Annals of the New York Academy of Sciences 1995771 485-490.

42 Achenbach TM, McConaughy SH \& Howell CT. Child/adolescent behavioral and emotional problems: implications of crossinformant correlations for situational specificity. Psychological Bulletin $1987 \mathbf{1 0 1} 213-232$.

43 Cole DA, Hoffman K, Tram JM \& Maxwell SE. Structural differences in parent and child reports of children's symptoms of depression and anxiety. Psychological Assessment 200012 174-185.

44 Kessler RC, McGonagle KA, Nelson CB, Hughes M, Swartz M \& Blazer DG. Sex and depression in the National Comorbidity Survey. II: Cohort effects. Journal of Affective Disorders 199430 15-26.
45 Offord DR, Boyle MH \& Jones BR. Psychiatric disorder and poor school performance among welfare children in Ontario. Canadian Journal of Psychiatry 198732 518-525.

46 Jane MC, Canals J, Ballespi S, Vinas F, Esparo G \& Domenech E. Parents and teachers reports of DSM-IV psychopathological symptoms in preschool children: differences between urbanrural Spanish areas. Social Psychiatry and Psychiatric Epidemiology 200641 386-393.

47 Cohen J. A power primer. Psychological Bulletin 1992112 155-159.

Received 7 May 2008

Accepted 10 June 2008 